\title{
Culture and Stigma of Mental Illness: Path Analysis Conducted with Amos in Transcultural Psychiatry in Australia
}

\author{
Tan Kan Kuㄹ, Michael Ha ${ }^{2}$ \\ ${ }^{1}$ Victoria University Institute of Technology (VUIT), St. Albans Campus, Melbourne, Australia \\ ${ }^{2}$ Financial Mathematics Programme, Department of Mathematical Sciences, Xi'an Jiaotong-Liverpool University, \\ Suzhou, China \\ Email: tankanku@gmail.com, Tan.Ku@vu.edu.au, Michael.Ha@xjtlu.edu.cn
}

Received April 2015

\section{Abstract}

Aim: The study was designed to elucidate the relationships between cultural values, contact level, differential nursing practices and stigma towards mental illness among General and Psychiatric Nurses ( $\mathrm{N}=208$ ) from Anglo and Chinese backgrounds in Australia. The study results aimed at informing education disciplines, health care providers and policy makers to examine strategies that seek to diminish stigma of mental illness and to reflect on cultural sensitive issues associated with mental illness. This paper reports analyses conducted with AMOS- 5 within the Chinese sample $(\mathrm{n}=$ 84). Method: A cross-sectional survey explored a number of factors such as contact factor, cultural values (Individualism (IND), In group Role Concern (IRC) and In group Interdependence (INT)), "General Stigma" measured by the Social Distancing (STPP) and Negative Stereotyped (DISL) Scales and how these factors might translate into nurses' endorsement of differential care practices between two vignettes (diabetes versus mental illness), which was termed "Practice Stigma". "Practice Stigma" was measured by Caring Approach (CARE), Nursing Satisfaction (SATI), Authoritarian Stance (AUTH) and Negativity (NEGA) Scales. Differential practices (DIF_CARE, DIF_SATI, DIF_AUTH and DIF_NEGA) were obtained by subtracting the diabetes case from the mental illness case. Findings: Chinese nurses endorsed more highly collectivist values measured by the variables "In group Interdependence" and "In group Role Concern" when compared with Anglo-Australian nurses, but there was no difference in individualist values measured by "Individualism". Chinese nurses endorsed more highly general stigma towards mental illness than Anglo nurses when statistically controlled for differences in background demographics and contact factors. Chinese nurses endorsed more care/support and authoritarianism in their clinical approaches than Anglo-Australian nurses, although there was no significant interaction effect between ethnicity and patient type on care and authoritarianism. Chinese nurses endorsed more highly differential negativity than Anglo nurses for the mental illness case than the diabetes case, an effect mediated by differences in general stigma between these two ethnic groups. Within the Chinese sample, higher contact was related to lower differential negativity for the mental illness case than the diabetes case. Several path analyses suggested that Chinese values influenced differential negativity, mediated by general stigma and prior diversified contact with people having a mental illness. 


\section{Keywords}

\section{Culture, Stigma, Mental Illness}

\section{Introduction}

\subsection{Background}

Psychiatric nursing is a specialized field of nursing which focuses on meeting the "mental health needs of the consumer, in partnership with family, significant others and the community in any setting" (Australian \& New Zealand College of Mental Health Nurses (ANZCMHN) [1] p. 3). Differences in health workers' attitudes are influenced by a variety of factors, such as the workers' beliefs and perceptions about mental illness, patients' characteristics, mental health training, and exposure through the interpersonal environment (whether social or through work setting) [2]. It is highly suggestive that such attitudes are also related to culture and that stigma towards mental illness is more widespread among the Chinese than their Anglo counterparts. For instance, Chinese people endorse authoritarian and restrictive attitudes towards those with mental illness [3] [4].

\subsection{Literature Review}

International studies indicated that increased contact with people having a mental illness was the best means for lowering stigma towards them in comparison with having knowledge in mental illness and protesting against society that condemned people with a mental illness [5]-[7]. "Culture" may have been a hidden variable in assessing attitudes with national contrasts of ethno-cultural groups. Several commentaries suggest that stigma is more severe in Asian, particularly, among the Chinese [8] [9].

\section{Methods}

\subsection{Subjects}

Two hundred and eight nurses participated in the study. Subjects were recruited by the snowballing technique and composed of nurses [83 Anglo Psychiatric (male $=31$, female $=52)$, 41 Anglo General $($ male $=3$, female $=$ 38), 49 Chinese Psychiatric (male $=25$, female $=24$ ) and 35 Chinese General (male $=1$, female $=34$ )] practicing in Melbourne, Australia. Age ranged from 21 to 65 years. Table 1 shows the sample demographics.

\subsection{Procedure}

Recruitment of participants was achieved through a snowballing technique. After ethics approval for the study was granted by the University of Melbourne Human Research Ethics Committee (HERC No. 020030), an initial pool of nurses $(n=20)$ of Chinese-Australian and Anglo-Australian backgrounds working in various health settings were identified and asked to participate in the study. This initial pool of nurses, known to the researcher $(\mathrm{Ku})$, was asked to approach potential participants and ask permission for the researcher to speak with them to introduce the study formally. Those nurses who indicated an interest in participating were asked to meet with the researcher for the purpose of explanation of the nature, purpose and procedure of the study. All participants signed written consent to anonymous participation. Data were collected in the latter part of 2002 and early 2003.

\subsection{Response Rate}

Three hundred and forty (347) surveys were disturbed. Two hundred and eight nurses out of 331 relevant participants returned the survey giving a $63 \%$ response rate $(208 / 331 \times 100)$.

\subsection{Statistical Analysis}

Principal component analyses were conducted on items to develop subscales related to cultural values, contact types, general and practice stigma. Analyses of variance and covariance were conducted to examine differences between nurse group and ethnicity and respectively, to account for possible differences in background demo- 
Table 1. Characteristics of the sample $(\mathrm{N}=208)$.

\begin{tabular}{cccccc}
\hline & $\begin{array}{c}\text { Chinese } \\
\text { Psychiatry } \\
(\mathrm{n}=49)\end{array}$ & $\begin{array}{c}\text { Chinese } \\
\text { General } \\
(\mathrm{n}=35)\end{array}$ & $\begin{array}{c}\text { Anglo } \\
\text { Psychiatry } \\
(\mathrm{n}=83)\end{array}$ & $\begin{array}{c}\text { Anglo } \\
\text { General } \\
(\mathrm{n}=41)\end{array}$ & $\chi^{2} / \mathrm{F}$ value \\
\hline Age $^{\#}$ (mean, s.d.) & $50.3(5.8)$ & $47.9(6.0)$ & $43.1(9.6)$ & $39.5(11.6)$ & $13.2^{* * * *} \mathrm{~F}(1,197)$ \\
Sex (male/female) & $25 / 24$ & $1 / 34$ & $31 / 52$ & $3 / 38$ & $35.4^{* * * *}$ \\
\hline
\end{tabular}

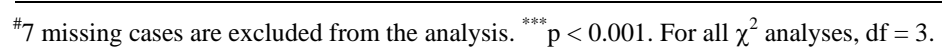

graphics, contact level and in the case of practice stigma, general stigma. The key findings revealed differences according to nurse type and ethnicity in several of the subscales. Psychiatric nurses endorsed a higher level of contact than general nurses with people who have a mental illness on the variables "Contact Through Work Situation", "Patient Help Nurses" and "External Socialisation with Patient", but not on the variable "Relative With Mental Illness". By virtue of more contact, psychiatric nurses also endorsed less general stigma, assessed by results from analyzing social distancing, but not by negative stereotyping of people with mental illness. With respective to practice stigma, while care and satisfaction did not differ according to patient type and nurse group, psychiatric nurses expressed less authoritarianism and negativity than general nurses towards the mental illness case than general nurses while lesser differences between nurse type were evident for the diabetes case. Statistical Package for Social Sciences (SPSS) Version 12 was used for statistical analysis.

\section{Results}

\subsection{General Stigma, Cultural Values and Contact Factors among Chinese and Anglo Nurses $(\mathrm{N}=208)$}

Table 2 indicates that General Stigma was related to cultural values and partially related to contact factors (that is, more contact, whether via work situation, or other means such as having a relative with mental illness or external socialization with someone with a mental illness) reduced stigma. However, contact factor did not influence stereotyping attitudes towards someone with a mental illness. Cultural values were related to general stigma.

\subsection{Contact Factors and Practice Stigma among Chinese and Anglo Nurses $(\mathrm{N}=208)$}

While Table 2 indicates the association between contact and general stigma, Table $\mathbf{3}$ indicates the association with practice stigma as represented by differential scores in nursing approaches. Contact, and especially measured by CWS and ESP, was negatively correlated with differential authoritarian stance and negativity. That is, the latter forms of nursing approaches were less differentiated between the cases of mental illness and diabetes as respondents tended to endorse higher levels of contact.

\subsection{Cultural Values, Contact, General Stigma and Practice Stigma among Chinese Nurse $(\mathrm{n}=84)$}

This section examines the relationship between Chinese cultural values, (IRC and INT), general stigma (social distancing and negative stereotyping) and differential negativity (DIFF_NEGA). In addition it is of interest to examine the influences of level of contact with the mentally ill and nurse type (general and psychiatric) on these relationships.

Path Analyses conducted with AMOS-5 [10] within the Chinese sample indicating significant paths between Chinese values (IRC and INT) and differential negativity, but mainly mediated by social distancing. Contact level was a mediator between social distancing and differential negativity. Negative stereotyped was directly positively associated differential negativity. The relationship between Chinese cultural values (IRC and INT), general stigma (STPP and NEGA) and differential negativity was examined further.

As a starting point, correlations among these measures were mapped as shown in Figure $\mathbf{1}$ in a provisional model that treats contact level as a possible influence on general stigma measures in addition to Chinese cultural values, and explores direct and indirect relationships between these and differential negativity. As shown in Figure 1, nurse type and contact level (with people having a mental illness) was highly correlated, sharing about 
Table 2. Pearson correlations between general stigma and other measures $(\mathrm{N}=208)$.

\begin{tabular}{ccc}
\hline CWS & $\begin{array}{c}\text { Social Distancing } \\
\text { (STPP Score) }\end{array}$ & $\begin{array}{c}\text { Negative Stereotyping } \\
\text { (DISL Score) }\end{array}$ \\
PHN & Contact Factors & $-0.08 \mathrm{~ns}$ \\
RMI & $-0.39^{* * *}$ & $-0.07 \mathrm{~ns}$ \\
INT & $-0.30^{* * *}$ & $0.04 \mathrm{~ns}$ \\
IRC & $-0.14^{*}$ & $0.21^{* *}$ \\
IND & Cultural Values & $0.25^{* * *}$ \\
& $0.52^{* * *}$ & $0.17^{*}$ \\
DIF_CARE & $0.45^{* * *}$ & $0.06 \mathrm{~ns}$ \\
DIF_SATI & $0.24^{* * *}$ & $0.18^{* *}$ \\
DIF_AUTH & Nursing Approaches & $0.15 \mathrm{~ns}$ \\
DIF_NEGA & $0.03 \mathrm{~ns}$ & $0.28^{* * *}$ \\
\hline
\end{tabular}

${ }^{*} \mathrm{p}<0.05,{ }^{* *} \mathrm{p}<0.01,{ }^{* * *} \mathrm{p}<0.001 ;$ ns, not significant. CWS = Contact through Work Situation; PHN = Patient Helps Nurse; RMI = Relative with Mental Illness; ESP = External Socialisation with Patient; INT = Ingroup Interdependence; IRC = Ingroup Role Concern; IND = Individualism; DIF_CARE = Differential Caring Approach; DIF_SATI = Differential Nursing Satisfaction; DIF_AUTH = Differential Authoritarian Stance; DIF_NEGA = Differential Negativity.

Table 3. Pearson correlations between contact factors and practice stigma $(\mathrm{N}=208)$.

\begin{tabular}{cccccc}
\hline & CWS & PHN & RMI & ESP & TOTAL CONTACT \\
\hline DIF_CARE & $-0.10 \mathrm{~ns}$ & $-0.04 \mathrm{~ns}$ & $-0.03 \mathrm{~ns}$ & $-0.08 \mathrm{~ns}$ & $-0.08 \mathrm{~ns}$ \\
DIF_SATI & $0.05 \mathrm{~ns}$ & $0.12 \mathrm{~ns}$ & $0.13 \mathrm{~ns}$ & $0.07 \mathrm{~ns}$ & $0.12 \mathrm{~ns}$ \\
DIF_AUTH & $-0.18^{* *}$ & $-0.06 \mathrm{~ns}$ & $0.07 \mathrm{~ns}$ & $-0.22^{* *}$ & $-0.15^{*}$ \\
DIF_NEGA & $-0.26^{* * *}$ & $-0.06 \mathrm{~ns}$ & $-0.02 \mathrm{~ns}$ & $-0.26^{* * *}$ & $-0.23^{* *}$ \\
\hline
\end{tabular}

${ }^{*} \mathrm{p}<0.05,{ }^{* *} \mathrm{p}<0.01,{ }^{* * *} \mathrm{p}<0.001$; ns, not significant. CWS = Contact through Work Situation; PHN = Patient Helps Nurse; RMI = Relative with Mental Illness; ESP = External Socialisation with Patient; DIF_CARE = Differential Caring Approach; DIF_SATI = Differential Satisfaction; DIF_AUTH = Differential Authoritarian Stance; DIF_NEGA = Differential Negativity.

$80 \%$ of their variance. Thus, given the importance of contact in the literature and the strong co-variation with nurse type, the latter was not considered further in modeling the relationships among the variables. Contact level is evidently uncorrelated with Chinese cultural values, nor are cultural values directly related to differential negativity. Contact level is strongly negatively correlated with social distancing but not with negative stereotyping (despite these two being correlated), and it is correlated negatively with differential negativity. IRC is correlated with social distancing but not with negative stereotyping while INT is correlated with both general stigma measures. Higher endorsement of these values (which are significantly correlated) is associated with higher endorsement of stigma towards the mentally ill. Stigma, in turn, seems positively correlated with differential negativity although the correlation between social distancing and negativity only approached significance $(\mathrm{p}=0.056)$. Contact level was also correlated negatively with differential negativity.

In Figure 2, non-significant correlations are removed for clarity. In addition, given the correlation between the two values a factor score based on the two values appears in their place. The score was derived by principal components analysis showing one dimension, termed here "Chinese Values", that explained $71.28 \%$ of the scale scores variance. As seen in Figure 2, Chinese Values, as a measure, behaves similarly to the items in showing positive relationships with stigma indicators and no relationships with contact level and differential negativity. 


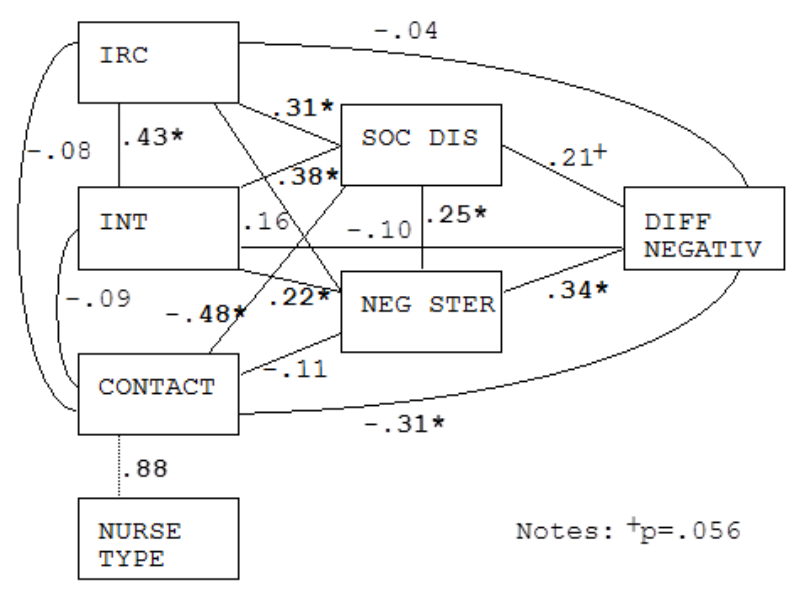

Figure 1. Representation of correlations.

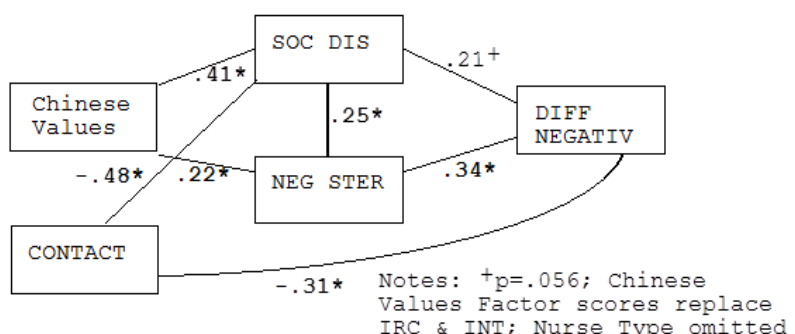

Figure 2. Representation of significant correlations.

(Nurse type is omitted in Figure 2 for reasons outlined earlier).

Figure 3 shows results of path analysis conducted with AMOS-5 [10]. Preceding this outcome a previous model with contact level excluded from the analysis indicated significant paths between Chinese Values and negative stereotyping, and the "weak" path between social distancing and differential negativity. However, once contact level was entered in the analysis these paths were no longer significant. Although the model shows good fit statistics to the data the relationships shown in Figure 3 suggest that contact level could be considered as a mediator of the relationship between social distancing and differential negativity.

Figure 4 shows the model specifying contact level as a mediator between social distancing and differential negativity, with only slight changes in fit statistics. As argued earlier the correlation between social distancing and negative stereotyping was small enough (though significant) to warrant keeping them as separate measures of general stigma. In the model in Figure 5 they are treated as independent, and in doing so, Chinese Values are seen to be associated with negative stereotyping. No additional paths were indicated.

The model of Figure $\mathbf{5}$ was re-expanded by including the measurement model for Chinese Values and this is shown on the left part of Figure 6. That is, Chinese Values is estimated as a latent variable based on IRC and INT and these are analysed together with the rest of the path model. As shown, there is a slight increase in path coefficients between Chinese Values and general stigma indicators but no other changes. The overall model fit is well within acceptable limits. However, examination of modification indices suggested one additional path, between INT and differential negativity. This is shown in the model represented in Figure 7, though the additional path was barely significant $(\mathrm{p}=0.046)$. Of interest, the relationship is negative, that is, higher endorsement of INT is associated with lower differential negativity, in the presence of the latent variable Chinese Values in the model.

Regardless of the details of the various alternative models, which are essentially similar in fit, the main general results suggest that Chinese cultural values are related to differential negativity, but mainly indirectly, that is, mediated by stigma and especially social distancing. Second, while contact level was expected to influence social distancing and negative stereotyping, given the causal association given to it in the literature in reducing stigmatizing attitudes, the results suggest that it is better placed as a mediator between social distancing and dif- 


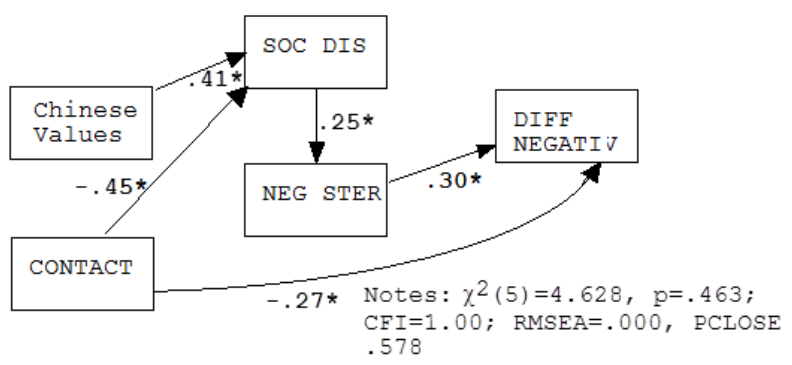

Figure 3. Path analysis panel B Model.

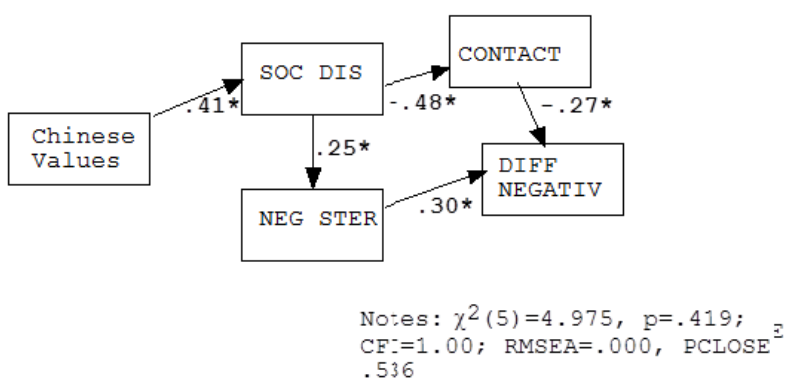

Figure 4. Path analysis respecified Model 1.

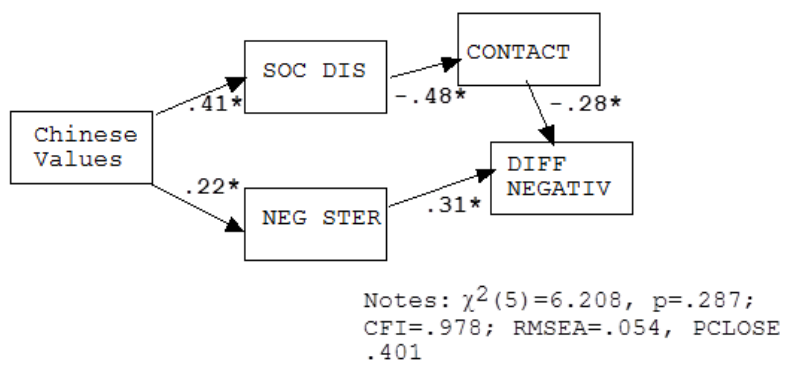

Figure 5. Path analysis respecified Model 2.

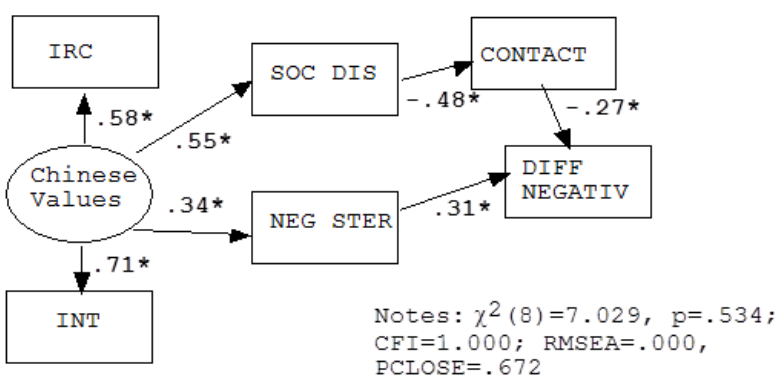

Figure 6. Path analysis respecified Model 3.

ferential negativity. Negative stereotyping is not mediated by contact level but is directly positively associated with differential negativity.

\section{Discussion}

This study was guided by three main results from the literature. First, the literature suggested contact with people having a mental illness should be associated with more moderate attitudes towards them. The present study compared psychiatric and general nurses on their general attitudes towards the mentally ill using two 


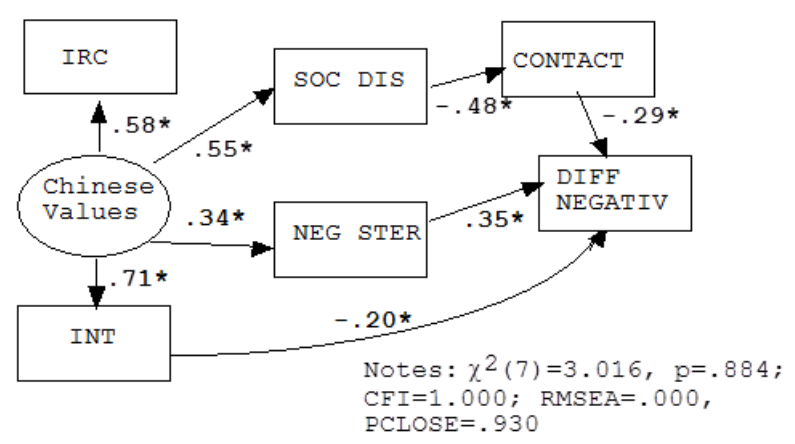

Figure 7. Path analysis respecified Model 4.

measures of general stigma derived in the course of the study. One focused on social distancing, stigma towards psychiatric patients (Social Distancing Scale, STPP) and the other on negative stereotyping (DISL). As expected, psychiatric nurses by virtue of their experience and perhaps specialty choice should endorse more contact and less stigma relative to general nurses.

Second, it was assumed in the literature that general stigma translated to practice stigma as represented by approaches to clinical care of people having mental illness. However, apart from Brinn's [11] and Ellsworth's [12] studies, this assumption had not been widely tested. In the present study the Nursing Relationship Scale (NRS) [13] was specifically developed to compare relative nursing care approaches endorsed for a case with mental illness versus a case with diabetes. The former case did attract more negative and less positive practice approach ratings [13].

Third, there was suggestion in the literature that stigma attached to mental illness might be subject to cultural indices. However, the relationship between culture and stigma was poorly understood. Thus, it was expected that within the Chinese group, if "culture" underpinned differences in stigma endorsement, then there would be an association between cultural values endorsement and measures of general and practice stigma.

In the present study, Chinese and Anglo-Australian nurses were compared on general and practice stigma measures. Given the possibility of acculturation among the Chinese, the study also explored whether this Chinese group differed from Anglo-Australians on indicators of collectivist and individualist values endorsement. If the expectation that differences in values was borne out by the results, then any attributions of stigma endorsement differences between ethnic group were due to "culture" would be strengthened.

\section{Conclusion}

The results support the notion that "culture" influences the stigma towards mental illness and has an effect on endorsement of differential nursing practice towards an individual with a mental disorder, particularly among Chinese nurses practicing in Australia. Aspect of the Chinese values specifically correlating with general stigma remains to be researched for future exploration.

\section{Competing Interests}

The authors declare that they have no competing interests.

\section{Authors' Contributions}

The first author carried out the study as part of a Master program at the Centre for International Mental Health (CIMH), The University of Melbourne under the supervision of the late Steven Klimidis, with co-supervision from Associate Professor Harry Minas, Director at CIMH. The first author prepared the first draft of the paper and the second author verified statistical results and edited the paper. Both authors have approved the final version of the manuscript.

\section{Acknowledgements}

The authors would like to thank Xian Jiaotong-Liverpool University and Victoria University Institute of Technol- 
ogy for supporting and sponsoring the publication of this paper. The first author acknowledges the substantial intellectual contribution of the late Steven Klimidis to the formulation of the study and data analyses reported in this paper.

\section{References}

[1] Australian \& New Zealand College of Mental Health Nursing (1995) Standards of Practice. Australian \& New Zealand College of Mental Health Nurses Inc., Greenacres, South Australia.

[2] Good, L.T., Berenaum, H. and Nisenson, L.G. (2000) Residential Caregivers Attitudes towards Seriously Ill Persons. Commentary Psychiatry, 63, 23-33.

[3] Shokoohi-Yekta, M. and Retish, P.M. (1991) Attitudes of Chinese and American Male Students towards Mental Illness. International Journal of Social Psychiatry, 37, 192-200. http://dx.doi.org/10.1177/002076409103700306

[4] Fan, C. and Karnilowicz, W. (2000) Attitudes towards Mental Illness and Knowledge of Mental Health Services among the Australian and Chinese Community. Australian Journal of Primary Health-Interchange, 6, 38-48. http://dx.doi.org/10.1071/PY00017

[5] Corrigan, P.W., River, L.P., Lundin, R.K., Penn, D.L., Uphoff-Wasowski, K., Campion, J., Mathisen, J., Gagnon, C., Bergman, M., Goldstein, H. and Kubiak, M.A. (2001) Three Strategies for Changing Attributions about Severe Mental Illness. Schizophrenia Bulletin, 27, 187-195. http://dx.doi.org/10.1093/oxfordjournals.schbul.a006865

[6] Corrigan, P.W. and Matthews, A.C. (2003) Stigma and Disclosure: Implications for Coming out of the Closet. Journal of Mental Health, 12, 235-248. http://dx.doi.org/10.1080/0963823031000118221

[7] Couture, S.M. and Penn, D.L. (2003) Interpersonal Contact and the Stigma of Mentalillness: A Review of the Literature. Journal of Mental Health, 12, 291-305. http://dx.doi.org/10.1080/09638231000118276

[8] Lin, T. (1983) Psychiatry and Chinese Culture. The Western Journal of Medicine, 139, 862-867.

[9] Ng, C.H. (1997) The Stigma of Mental Illness in Asian Cultures. Australian and New Zealand Journal of Psychiatry, 31, 382-390. http://dx.doi.org/10.3109/00048679709073848

[10] Arbuckle, J.L. (2003) AMOS-5 (Software). Smallwaters Core, Chicago.

[11] Brinn, F. (2000) Patients with Mental Illness: General Nurses’ Attitudes and Expectations. Nursing Standard, 14, 3236. http://dx.doi.org/10.7748/ns2000.03.14.27.32.c2792

[12] Ellsworth, R.B.A. (1965) A Behavioural Study of Staff Attitudes toward Mental Illness. Journal of Abnormal and Social Psychology, 70, 194-200. http://dx.doi.org/10.7748/ns2000.03.14.27.32.c2792

[13] Ku, T.K. and Minas, I.H. (2010) Development of the Nursing Relationship Scale: A Measure of Interpersonal Approaches in Nursing Care. International Journal of Mental Health System. www.ijmhs.com/contents/4/12 http://dx.doi.org/10.1186/1752-4458-4-12 